\title{
Control de autoridades en catálogos en línea
}

\author{
ANA M. MARTÍNEZ* \\ NORMA E. MANGIATERRA** \\ CRISTINA RISTUCCIA** \\ MARIANA PICHININI* \\ MÓNICA G. PEN É* \\ ${ }^{*}$ Cátedra de Clasificación I y II. \\ ${ }^{* *}$ Cátedra de Catalogación I. \\ D epartamento de Bibliotecología. Facultad de Humanidades y \\ Ciencias de la Educación. Universidad Nacional de La Plata. \\ Calle 48 e/ 6 y 7-5o piso, 1900 La Plata, Argentina. \\ E-mail (AMM): ammarti@ netverk.com.ar \\ E-mail (NEM): biblos2@ satlink.com.ar
}

\section{RESUMEN}

Se revisa la literatura especializada sobre el control de autoridades en los catálogos en línea, incluyendo sus definiciones, funciones, gestión y tendencias actuales, así como la situación en bibliotecas de América Latina. Si bien no se trata de una práctica nueva, ha sido revalorada a partir del alto índice de fracasos (cero registros recuperados) detectado durante las búsquedas en línea realizadas por los usuarios en los catálogos de biblioteca. Las principales ventajas del control de autoridades son: a) mejora la precisión y la reall orientando al usuario durante la búsqueda mediante un sistema de referencias cruzadas, b) en la recuperación ofrece un $100 \%$ de garantía documental, c) mejora y agiliza los procesos técnicos, incluyendo la verificación, validación y cambios globales en el catálogo y d) ahorra espacio de almacenamiento. Las desventajas son el alto costo y el esfuerzo intensivo que demanda la implementación y mantenimiento de las listas de autoridades. En el futuro inmediato se apunta a desarrollar la tecnología, incrementar la productividad, promover la cooperación nacional e internacional y realizar más investigaciones. En América Latina se necesita discutir el control de autoridades en profundidad, especialmente en relación con los sistemas de gestión bibliotecaria desarrollados en la región, el muy difundido sattware CDS/ ISIS, diversos formatos no MARC para datos bibliográficos, en especial aquellos distribuidos por organismos internacionales (por ejemplo CEPAL, LILACS, AGRIS, INIS, etcétera), el relevamiento de las listas de autoridades ya existentes, la compilación de listas nacionales, la cooperación nacional y regional, la capacitación profesional y el compromiso que deben asumir al respecto las bibliotecas nacionales.

\section{ABST RACT}

The literature on authority control of on-line catalogs is reviewed, including its definitions, functions, management, and current trends, as well as the present situation in Latin America. Although authority control is not a new practice, it has been revalued due to the high

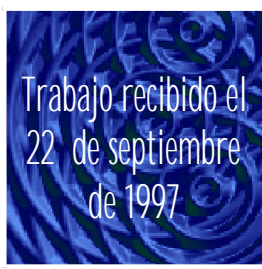


failure index (zero records retrieved) detected during the online searches of library catalogs carried out by users. The main advantages of authority control are: a) improvement of precision and recall, guiding the user during the search with a cross reference system, b) guarantee of $100 \%$ document retrieval, c) better and faster technical processes, including verification, validation and global changes in the catalogs, d) savings in storage space. The disadvantages include the high cost and intense effort required for implementing and maintaning authority files. The immediate future aims at technological improvements, productivity increase, national and international cooperation, as well as more research development. In depth authority control discussions are necessary in Latin America, particularly related to: library management systems developed in the region, the widely used software CD S/ ISIS, several non-MARC formats for bibliographic data specially those provided by international organizations (e.g. CEPAL, LILACS, AGRIS, INIS, etc.), the identification of already existing authority lists, the compilation of national lists, national and regional cooperation, professional continuing education, and the commitment of national libraries.

\section{INTRODUCCIÓN}

F l control de autoridades es el proceso de mantener la coherencia de la forma $\perp$ verbal usada para representar un punto de acceso - sea nombre, título uniforme 0 materia- en un catálogo de biblioteca y mostrar además las relaciones entre dichos puntos de acceso (Clack, 1985; Taylor, 1989 y 1992; Tillet, 1989). Este proceso se concreta en una serie de operaciones que permiten elaborar un asiento o registro de autoridad; el conjunto de estos registros se denomina lista deaur tonidades (ISO 5127/3a, 1981; UNE 50113/3a, 1991) y puede ser de nombres (personas, entes corporativos, conferencias, familias, lugares geográficos), de títulos uniformes (monográficos y de series) o de materias (epígrafes o descriptores). Un nombre, un título uniforme 0 una materia, establecido oficialmente como punto de acceso autorizado, constituye una autoridad

El control de autoridades no es una práctica nueva, ya que a fines del siglo pasado, Charles A. Cutter prescribió la elección de un único encabezamiento como punto de acceso, con remisiones desde las formas variantes y relacionadas, que debían constituir un sistema de conexiones o referencias cruzadas. A partir de allí, la lista de autoridades se convirtió en un instrumento de apoyo para los procesos técnicos, pero con el tiempo muchas bibliotecas la sustituyeron por la consulta en el catálogo público y el marcado de la lista de epígrafes o del tesauro (Taylor, 1992). Sin embargo, desde la aparición de los catálogos en línea, se ha instalado un amplio debate sobre el control de autoridades, sus ventajas, desventajas y desarrollos futuros. Por tal motivo, en el presente trabajo, intentamos revisar la bibliografía sobre el tema y presentar un estado del arte que abarque 
aspectos tales como definiciones, funciones, gestión (operaciones, normalización, tecnología y cooperación), así como proponer una discusión sobre el control de autoridades en bibliotecas de América Latina.

\section{FUNCIONES DEL CONTROL DE AUTORIDADES}

El control de autoridades permite mejorar dos funciones del catálogo de biblioteca, que fueron definidas por el propio Cutter (Jáudenes Casaubón, 1996; Taylor, 1992; Tillet, 1989; Y ounger, 1995a,b):

- Función de identificación, es decir, la posibilidad de encontrar un documento particular, entre todos los que forman la colección, a partir del uso de formas coherentes de los puntos de acceso de autor, título o materia.

- Función de reunión, es decir, la posibilidad de encontrar todos los documentos de un mismo autor, título uniforme o materia que forman la colección.

Estas funciones se pueden asimilar a los conceptos de precisión -capacidad de limitar una búsqueda- y reall -capacidad de ampliar una búsqueda (Younger, 1995a,b). En la indización, los vocabularios controlados mejoran la precisión mediante el control de homónimos y polisemas y la construcción de términos compuestos, mientras que mejoran la reall mediante el control de sinónimos y las relaciones asociativas (Aitchison y Gilchrist, 1987; Dubois, 1987; Lancaster, 1995; Svenonius, 1986). Estas propiedades son, por supuesto, aplicables a la lista de autoridades de materia, ya que ésta constituye en realidad el vocabulario controlado de una biblioteca en particular. Sin embargo, los conceptos de precisión y reall también son aplicables a la lista de autoridades de nombres y títulos, para cuyo control se siguen las reglas de AACR2R y las interpretaciones de esas reglas (Gorman y Winkler, 1988; Library of Congress, 1990). Así, la adición de fechas de nacimiento y muerte 0 las aclaraciones de iniciales entre paréntesis contribuyen a mejorar la precisión, mientras que las referencias que conectan los nombres autorizados y sus formas variantes o los diferentes títulos de una misma obra y su título uniforme contribuyen a mejorar la reeall (Taylor, 1992).

De modo que los ejemplos 1.1 a 1.10 de la Tabla 1 aumentan la precisión y los ejemplos 2.1 a 2.3 y 3.2 a 3.3 aumentan la reeall, mientras que 2.4 , 3.1, 3.4, 4.1 y 4.2 aumentan la precisión si se elige una sola opción y aumentan la reall si se seleccionan varias o todas las opciones.

Ahora bien, diversos estudios sobre el desempeño de los usuarios durante la búsqueda en catálogos en línea, realizados principalmente en Estados Unidos, han demostrado altos índices de fracaso que alcanzan 30-40\% de búsquedas con cero registros recuperados para autor o título y 40-50\% para materia (Borgman, 1996; Ríos García, 1991; Shiao-Feng, 1994). 


\section{Tabla 1}

Ejemplos de registros de autoridad, registros de referencia específica y registros de referencia general

\section{Ejemplo 1 \\ Diferenc iación de homónimos y \\ polisemas para nombresy \\ materias:}

1.1 Gutiémez, M.F. (Manuel FrancisCo), 1910-1991

1.2 Gutiérez, M.F. (Manuel FrancisCo), 1940-

1.3 Gutiémez, M.F. (Mańa Florencia), 1965-

1.4 Córdoba (Argentina : ciudad)

1.5 Córdoba (España : ciudad)

1.6 Córdoba (México : ciudad)

1.7 Congreso Latinoamericano de Bioquímica Clínica (12 : 1995 : Buenos Aires)

1.8 Congreso Latinoamericano de Bioquímica Clínica (13 : 1997 : Caracas)

1.9 Langosta (Crustáceo)

1.10 Langosta (Insecto)

\section{Ejemplo 2}

Registro de autoridad de nombres, títulos uniformes y materia

2.1 Almafuerte

x Palacios, Pedro Bonifacio

2.2 OEA

xOrganización de Estados Americanos

2.3 Tragedia de Hamlet, Pŕncipe de Dinamarca

UP Hamlet
2.4 Contabilidad

TR Contadores públicos

Ejemplo 3

Registro de referencia específica de nombres, tútulos uniformes y materia

3.1 Escardó, Florencio

Para lasobrasde este autorbajo su seudónimo véase además Piolín de Macramé

3.2 Organización de las Naciones Unidas para la Educación, la Ciencia y la Cultura Véase UNESCO

3.3 El libro del pueblo de Dios Véase Biblia. N.T.

3.4 Danzas follónicas TG Danzas

\section{Ejemplo 4}

Registro de referencia general

4.1 Novelistas peruanos

Véase además el nombre de cada novelista individual, por ejemplo Vargas Losa, Mario

\subsection{Historiografía}

Se incluyen aquí las obras sobre la disciplina Histoniografía; para las obras sobre la historiografía particularde una disciplina, institución, país, etc. véase además la subdivisión Histoniografía bajo esos encabezamientos, por ejemplo Arte-Historiografía, Iglesia Católica-Histoniografía, Alemania-Historiografía, etcétera. 
Los principales errores detectados en la búsqueda por autor incluyen el nombre y apellido en orden directo y no invertido, errores de mecanografía y ortografía, truncamiento incorrecto, espaciamiento incorrecto, primera palabra incorrecta, demasiadas palabras solicitadas, persona como título o materia, intento de búsqueda booleana, etcétera (Bangalore, 1995; Peters, 1989; Sinnott, 1993). En cuanto a la búsqueda por materia, las principales dificultades se deben a errores de ortografía y mecanografía, búsquedas muy largas, pocos epígrafes o descriptores y una falta de coincidencia entre la terminología del usuario y la del sistema (Peters, 1989; Ríos G arcía, 1991; Shiao-Feng, 1994). Las investigaciones de Sinnott (1993) y Bangalore (1995) coinciden en que el control de autoridades reduce los índices de fracaso en la búsqueda por autor, con $26 \%$ y 13\% respectivamente, que contrastan con el 54\% informado por Hunter (1991) y el $31 \%$ de Peters (1989), en sistemas sin control de autoridades. Por su parte, Wilkes y Nelson (1995) llevaron a cabo una investigación en la que demostraron que la búsqueda por materia en un catálogo en línea sin control de autoridades ofrecía $59.4 \%$ de éxitos y $40.6 \%$ de fracasos; cuando estas últimas búsquedas fracasadas se repitieron en un catálogo con el mismo lenguaje de indización, pero con control de autoridades, se logró reducir el índice de fracaso a sólo 10\% de la muestra original.

Algunos autores consideran que para solucionar este alto índice de fracasos no es necesario el control de autoridades, sino la utilización de las poderosas técnicas de búsqueda con que cuenta la computadora (operaciones booleanas, truncamiento, adyacencia), así como la recuperación palabra por palabra (Shiao-Feng, 1994; Tillotson, 1995; Y ounger, 1995a,b). Watson y Taylor (1987) descubrieron que $82.8 \%$ de los nombres personales y $34 \%$ de los corporativos en la lista de autoridades Library of Congress NameAuthority File(LCNAF) eran innecesarios si se contaba con recuperación palabra por palabra y truncamiento a la derecha.

Sin embargo, los usuarios han demostrado un severo rechazo hacia la utilización de las técnicas de búsqueda. Peters (1989), de la University of Missouri, encontró que los usuarios aplicaban los operadores booleanos en menos del $1 \%$ de las búsquedas, mientras que Millsap y Ferl (1993), de la University of California at Santa Cruz, detectaron un 10\% y Olsen \& al. (1989), de la Brigham Young University, el 24\%. Pat Ensor (1992), de la Indiana State University, llevó a cabo una encuesta en la que el $40 \%$ de los encuestados ni siquiera pudo contestar si comprendía el uso de los operadores booleanos. De acuerdo con Borgman (1996), investigaciones realizadas en el área de la psicología cognitiva demuestran que las personas no aplican la lógica en su vida cotidiana, sino que siguen un juicio intuitivo en el que AND y OR se asumen en su sentido lingüístico, es decir, un AND inclusivo que amplía y un OR exclusivo que limita o reduce, exactamente al revés que los operadores booleanos. Además, Peters (1989) y Kern-Simirenko (1983) coinciden en señalar el escaso uso del truncamiento para comenzar una búsqueda, en tanto que Fidel (1991) y Hancock-Beaulieu (1990) han demostrado 
las dificultades que produce el desconocimiento de estas técnicas para reconvertir una búsqueda poco satisfactoria.

En cambio, la recuperación palabra por palabra aplicada a nombres, títulos, epígrafes o descriptores, notas de contenido, resúmenes, etcétera, ha sido una alternativa más exitosa, ya que permite recuperar fácilmente un punto de acceso verbal por cualquiera de las palabras que lo forman, sin importar el orden en que se encuentran (Ensor, 1992; Olsen, 1989). También incrementa los índices de precisión y reall, pero de diferente manera, ya que su nivel de especificidad mejora la precisión, mientras que la posibilidad de recuperar una palabra en todos los campos de la base de datos del catálogo mejora la reall (Aitchison y Gilchrist, 1987; Dubois, 1987; Lancaster, 1995; McJunking, 1995; Svenonius, 1986). Sin embargo, tiene también sus desventajas: por un lado aumenta los índices de sobrerrecuperación (más de 50 registros recuperados), como ha sido comprobado por Tillotson (1995), quien al analizar la recuperación palabra por palabra en dos catálogos en línea obtuvo un promedio de 1063 y 431 registros por búsqueda, respectivamente. Por otro lado, si el usuario solicita VASCONCELOS desde el menú de búsqueda, obtendrá los registros referidos a las obras escritas, entre otros, por el brasileño JoséMauro deVascondos o por el mexicano JoséV ascondos Caldaớn así como los estudios y biografías sobre ambos; si solicita COLERA recuperará registros referidos a esa enfermedad y a la novela El amor en los tiempos dd đedea; si solicita BANCOS, se encontrará con información sobre entidades financieras, muebles, cúmulos de arena, archivos de datos, etcétera.

D esde luego, la solución no consiste en elegir entre recuperación palabra por palabra 0 control de autoridades, sino en combinar ambos recursos en el mismo catálogo con el fin de ampliar las oportunidades de acceder a la información y de complementar las desventajas de una con las ventajas del otro y viceversa (D ubois, 1987; Svenonius, 1986; Tillet, 1996; Y ounger, 1995a,b).

En adición a lo anterior, es importante remarcar que la lista de autoridades de una biblioteca contiene los puntos de acceso que han sido utilizados en esa biblioteca durante la catalogación e indización, y nada más que esos puntos de acceso, de modo que cuando el usuario inicia la búsqueda en la lista de autoridades puede tener la certeza de que recuperará al menos un documento que responda a la autoridad elegida, es decir, obtendrá un 100\% de garantía documental (ninguna búsqueda con cero registros recuperados). 


\section{GESTIÓN DEL PROCESO DE CONTROL DE AUTORIDADES}

\section{0 peraciones}

El proceso del control de autoridades consiste en llevar a cabo una serie de operaciones que incluyen tanto el trabajo intelectual de establecer las autoridades y sus referencias, como las tareas de implementar, mantener y administrar la lista de autoridades (Clack, 1985; Jáudenes Casaubón, 1996; Taylor, 1992; Tillet, 1989). Básicamente son las siguientes:

1. Verificación de una autoridad: una vez identificado un posible punto de acceso como autor, título uniforme o materia, se debe consultar la lista de autoridades y controlar si ese punto de acceso ha sido aceptado como autoridad; si es así, se debe asignar esa autoridad coherentemente.

2. Creación de una autoridad: si por el contrario, el punto de acceso no coincide con una autoridad, ésta debe crearse y registrarse en la lista de autoridades.

3. Establecimiento de referencias cruzadas: una vez determinada la autoridad, deben establecerse las relaciones equivalentes (véase/ $\mathrm{x}$, Use/ UP) entre una autoridad y sus formas variantes, así como las relaciones jerárquicas (TG/TE) y asociativas (véase además/ Xx, TR/ TR).

4. Registro de autoridades y sus referencias cruzadas: la lista de autoridades en línea es una base de datos que debe definirse de acuerdo con un formato normalizado y un softwarede gestión apropiado.

5. Mantenimiento de la lista de autoridades: requiere una permanente revisión, verificación y validación del sistema para incorporar altas, modificaciones y bajas, actualizando no sólo las autoridades, sino también las referencias cruzadas y sus respectivas reciprocidades.

De acuerdo con las directrices de la International Federation of Library Associations (IFLA, 1984, 1993a,b y 1995), en una lista de autoridades se pueden incluir tres tipos de registros: los registros de autoridad, los registros de referencia específica y los registros de referencia general. Un registro de autoridad consta básicamente de: a) un encabezamiento de autoridad que constituye el punto de acceso autorizado y b) un trazado que incluye las referencias a las formas variantes no utilizadas (señaladas con los símbolos $\mathrm{x}, \mathrm{UP}, \leftarrow,<$ ) 0 a otros encabezamientos de autoridad relacionados (Vémse adamás, TR, $»,>><<$ ), como los ejemplos 2.1 a 2.4 de la Tabla 1 . Un registro de referencia específica puede ser de dos tipos: a) el encabezamiento de referencia específica es una forma variante que no se usa y remite a un encabezamiento de autoridad mediante los símbolos Véase, Use, $\rightarrow$, > como los ejemplos 3.2 y 3.3 de la Tabla 1 ; b) el encabezamiento de referencia específica es un encabezamiento de autoridad que remite a otro encabezamiento de autoridad, mediante los símbolos Véase adamás, TR, ««, $>><<$; en este caso, si se desea indicar una relación jerárquica, se pueden sustituir los símbolos anteriores por los correspondientes TT, TG, », >> $0 \mathrm{TE}, \ll<<y$ 
también es posible redactar estas referencias en forma de nota; los ejemplos 3.1 y 3.4 de la Tabla 1 corresponden a la referencia específica. Por último, un registro de referencia general incluye: a) un encabezamiento de referencia general, que suele ser un encabezamiento colectivo y b) los encabezamientos de autoridad a los que se remite mediante una nota explicativa o referencia sábana, como se muestra en los ejemplos 4.1 y 4.2 de la Tabla 1.

La planificación de un sistema de autoridades debe tener en cuenta distintos aspectos; en este sentido, resultan de gran utilidad las experiencias de diversas bibliotecas que han sido publicadas en la literatura especializada (Bechtel, 1992; Gibbs y Bisom, 1989; Goldman y Havens, 1990; Goldman y Smith, 1989; Halverson \&al, 1992; Irwin, 1993; Kirby, 1989; McCurley, 1993; Moreno y Quijano Solís, 1991; Park, 1992; Ridgeway, 1989; Santizo y Rezabek, 1992; Sullivan, 1992; Vela de la Sancha,1996).

Cuando una biblioteca decide implementar su propia lista de autoridades en línea, debe considerar el problema de la reconversión, que resulta imprescindible si se pretende lograr la vinculación entre la lista de autoridades y el catálogo en línea; se pueden presentar dos situaciones: que la biblioteca posea una lista de autoridades en ficha que pueda ser reconvertida al sistema en línea, o bien que la biblioteca no posea una lista de autoridades, pero quiera incluir en la nueva los puntos de acceso ya utilizados en su catálogo (Irwin, 1993; Maccaferri, 1992; McCurley, 1993; Wendler, 1995).

También es necesario decidir si las operaciones de implementación y reconversión de la lista de autoridades se realizarán en la biblioteca o se contratará una empresa catalogadora, en cuyo caso deben evaluarse los servicios y precios ofrecidos por cada proveedor (Johnston, 1989; Maccaferri, 1992; Wendler, 1995). En una encuesta realizada por Santizo y Rezabek (1992), 53\% de las bibliotecas había preferido contratar el trabajo con terceros, $12 \%$ lo hizo en la biblioteca y $32 \%$ combinó ambas opciones. Una vez implementada la lista y completada la reconversión, el personal de la biblioteca puede hacerse cargo de su mantenimiento.

D e acuerdo con Maccaferri (1992), la gestión de la lista de autoridades en línea no demanda mucho personal; por ejemplo en la Kent State University, de unas 12 personas que se requerían para mantener la lista de autoridades en fichas, se pasó a una sola persona responsable de la versión en línea. En la University of California at Los Angeles (UCLA), las operaciones de control de autoridades para el catálogo Orion (más de un millón de registros bibliográficos) han quedado a cargo de un bibliotecario y cuatro ayudantes. En la Sterling C. Evans Library de la Texas A\&M University, la sección de autoridades ha sido creada con las cinco personas que mantenían el catálogo en fichas, quienes recibieron entrenamiento en servicio para las operaciones pertinentes (Halverson \&al., 1992).

Las operaciones de control de autoridades son costosas y laboriosas; para OCLC (1995) constituyen el 30\% del costo total de los procesos técnicos, pero según Tillett (1989) alcanzan casi la mitad. Byrd y Sorury (1993), de la Indiana 
University, han calculado el costo de las operaciones de control de autoridades en US\$2.42 por registro, de modo que una biblioteca con cien mil puntos de acceso establecidos tendría que desembolsar aproximadamente un cuarto de millón de dólares para conformar su lista de autoridades.

En consecuencia, se discute si todos los puntos de acceso necesitan control de autoridades o sólo algunos. Idealmente, deberían establecerse autoridades para todos los puntos de acceso, pero en la práctica no siempre es posible. Diversos estudios intentan identificar criterios para esta selección de prioridades (Bogan, 1996; Calhoun, 1996; Fuller, 1989; Itoga \& al., 1996; McG rath, 1993; Y ounger, 1996). A manera de ejemplo, Bogan (1996) considera que para los nombres personales las prioridades serían: a) nombres usados como materia, b) apellidos compuestos, c) apellidos con prefijos, d) nombres de mujeres (solteras/ casadas), e) nombres de personas cuyo elemento de entrada no sea un apellido (monarcas, santos, papas, etc.), f) apellidos muy comunes (en inglés se han establecido cuatro: Jones, Johnson, Smith y Miller), g) nombres latinizados, h) nombres anteriores al siglo veinte, i) nombres con aclaración de iniciales o similares.

Una manera de proporcionar autoridades para todos los puntos de acceso y al mismo tiempo limitar el trabajo de autoridades es la informada por McG rath (1993) para las bibliotecas de la State University of New York at Buffalo, que se basa en la utilización del llamado registro de nivel mínimo o provisional. Este registro es creado por el sistema sin intervención humana, ya que cada punto de acceso utilizado en el catálogo en línea es verificado contra la lista de autoridades; si no se encuentra allí, el sistema automáticamente crea un registro mínimo o provisional que por lo general se limita al punto de acceso verificado, pero que en algunos casos puede incluir también algunas referencias (Anderson, 1991); luego, el bibliotecario de autoridades completa los registros que requieren referencias cruzadas, mientras que el resto se mantiene solamente con el nivel mínimo.

Los costos también se reducen cuando se accede a una lista de autoridades reconocida, por ejemplo las de Library of Congress. Aunque las listas son caras, hay distintas organizaciones como OCLC, RLIN, WLN, que hacen posible su consulta y la copia, no de todos los registros, sino sólo de aquellos que se necesitan (Dickson y Zadner, 1989). Maccaferri (1992) comenta la experiencia de UCLA, y señala que sólo $15 \%$ de los registros de autoridades fue original, mientras que 85\% se copió de otras listas.

De todas formas, las bibliotecas no siempre cuentan con personal suficiente y capacitado, ni presupuesto para llevar a cabo este control de autoridades y, en adición, muchas veces realizan un trabajo redundante e incompatible (Tillet, 1989). El resultado, entre otras cosas, es una productividad insuficiente de registros de autoridades: sólo el 50\% de los nombres personales y el 30\% de los entes corporativos, registrados como puntos de acceso en el catálogo colectivo de OCLC, se encontraban en las respectivas listas de autoridades de Library of Congress (OCLC, 1995). 


\section{NORMALIZACIÓN}

Al igual que el catálogo en línea, la lista de autoridades requiere un formato estándar que permita definir la base de datos para su gestión y al mismo tiempo intercambiar estos datos con otras bibliotecas (Jáudenes Casaubón, 1996; Taylor, 1992). En 1976, Library of Congress inició el desarrollo de su formato de autoridades, con una primera edición al año siguiente. Luego de profundas revisiones y modificaciones, se publicó una nueva edición en 1993, con el título USMARC format for authority data, que se actualiza periódicamente. Por su parte, IFLA ha desarrollado también su formato de autoridades UNIMARC / authorities Univesal format for authorities, con una primera edición en 1977 y la segunda y última en 1991. Además, existen formatos de autoridades nacionales como UKMARC, IBERMARC, etcétera (IFLA, 1991; Jáudenes Casaubón, 1995; Library of Congress, 1995a). Tanto UNIMARC como USMARC han establecido claramente la vinculación entre sus formatos de autoridades y los respectivos formatos bibliográficos, de modo que, por ejemplo, el campo correspondiente a nombre de persona en el formato de autoridades es compatible con el campo del formato bibliográfico para autor personal.

UNIMARC y USMARC se basan en AACR2R y tanto IFLA como Library of Congress han complementado sus formatos con varios instrumentos prescriptivos: IFLA publicó en 1984 sus Guiddines for authority records and entries(GARE) y en 1993 las Guiddines for subjet authrity records and entries (G SARE) - ambas traducidas en España-, mientras que Library of Congress cuenta con varios manuales que apoyan el control de autoridades (G orman y Winkler, 1988; IFLA, 1984, 1993a,b y 1995; Library of Congress, 1990, 1992, 1994, 1996a).

\section{TECNOLOGÍA}

Taylor (1992) distingue dos posibilidades para la lista de autoridades en línea: la lista implícita o separada del catálogo que presenta apenas algunas ventajas respecto a la lista en fichas y la lista explícita o vinculada con el catálogo, que desde luego constituye el aporte realmente valioso del control de autoridades en línea. En algunos sistemas, la lista comparte una misma base de datos con el catálogo, mientras que en otros se encuentra en bases de datos separadas, pero interconectadas.

La elección del software debe tener en cuenta el almacenamiento de los registros de autoridades, de acuerdo con los formatos aceptados internacionalmente, el mantenimiento de la lista de autoridades con sus adiciones, modificaciones y eliminaciones, la vinculación entre las autoridades y sus referencias cruzadas para obtener reciprocidades automáticas, la vinculación entre las autoridades y los registros del catálogo para facilitar tanto la tarea de almacenamiento como la de recuperación, verificación, validación y posibles cambios globales en el catálogo, la vinculación con otras listas de autoridades externas para poder importar y 
exportar registros de autoridades, diversas facilidades para la edición, exhibición, exploración, impresión y copia de la lista de autoridades, así como la generación de estadísticas e informes destinados a la evaluación y gestión del sistema (Aliprand, 1993; Anderson, 1991; Bechtel, 1992; Clack, 1990; Fiegen \&al., 1990; Fox y Kanafani, 1989; Gibbs y Bisom, 1989; Goldman y Havens, 1990; Goldman y Smith, 1989; Johnston, 1989; Kirby, 1989; Moreno Jiménez, 1992; Ridgeway, 1989; Sullivan, 1992; Vela de la Sancha, 1996; Wendler, 1995).

Como ya se mencionó, el software debe crear automáticamente los registros de autoridades provisionales o de nivel mínimo (Anderson, 1991; McGrath, 1993). Por otro lado, debe facilitar los permanentes cambios que se requieren para el mantenimiento tanto de la lista como del catálogo (Fox y Kanafany, 1989; Grady, 1996; Van Pulis, 1996). De acuerdo con Calhoun y O skins (1992), de las 1388 operaciones de control de autoridades que realiza Library of Congress en promedio por día, 60\% corresponde a nuevos registros, 38\% a modificaciones y $2 \%$ a bajas; esta relación se mantiene igual para los nombres y títulos, pero se invierte para materias: $38 \%$ de altas, $60 \%$ de modificaciones y $2 \%$ de bajas. Entonces, el sistema debe llevar a cabo la mayor cantidad de cambios globales; por ejemplo, en la reconversión del sistema Hollis de la Harvard University, el software empleado permitió realizar 1.6 millones de correcciones en los puntos de acceso de 5.3 millones de registros del catálogo, a partir de poco más de 684,000 registros de autoridades, en menos de año y medio, a razón de 60,000 registros transferidos quincenalmente, vía FTP, a la empresa catalogadora; las operaciones llegaron a realizarse a un ritmo de 25,000 correcciones por hora, sin intervención humana (Wendler, 1995).

O tro aspecto que debe considerarse es la interfaz usuario-sistema y la interfaz bibliotecario-sistema, ya que la lista de autoridades ha pasado a ser una herramienta útil tanto en la recuperación como en el almacenamiento de información. La interfaz debe ofrecerle al usuario la posibilidad de: a) explorar la lista de autoridades, sin tener que adivinar desde el menú de búsqueda qué términos se han utilizado para la descripción del documento, b) seleccionar una autoridad mediante un sistema orientador de referencias cruzadas y c) evitar errores de ortografía, mecanografía, orden de palabras, etcétera. Por ejemplo, si el usuario solicita el nombre MIGUEL ANGEL ASTURIAS, el sistema debe contestarle véaseASTURIAS, MGUEL ANGEL; el usuario se dirige a esta autoridad 0, mejor aún, es remitido automáticamente por el sistema; selecciona la autoridad con sólo presionar una tecla y se muestran en pantalla los registros documentales del catálogo que responden a la autoridad elegida (el usuario no necesita darse cuenta de que el sistema ha vinculado la lista de autoridades con el catálogo).

De manera similar, el bibliotecario de procesos técnicos que necesite agregar nuevos registros en el catálogo, en vez de mecanografiar los puntos de acceso, puede explorar la lista de autoridades orientándose con el sistema de referencias cruzadas, seleccionar la autoridad pertinente con sólo presionar una tecla y que 
esta autoridad se registre automáticamente en el campo correspondiente del catálogo en línea, evitando así errores de ortografía y mecanografía y agilizando la tarea de catalogación e indización.

D ebe destacarse también que el control de autoridades reduce la cantidad de términos controlados que debe manejar el índice invertido del catálogo en línea, ya que las formas variantes de un punto de acceso se almacenan una sola vez en la lista de autoridades, mientras que en el catálogo se almacena una única forma autorizada.

\section{COOPERACIÓN}

Al igual que otros aspectos de los procesos técnicos, tanto los costos como las tareas del control de autoridades se reducen si se comparten con sistemas interbibliotecarios. Entre las experiencias cooperativas se pueden mencionar el Program for Cooperative Cataloging (PCC), establecido por Library of Congress en 1995, integrado por más de 200 bibliotecas, que han acordado crear registros únicos, bajo formas mutuamente aceptadas y facilitar el uso de esos registros. El PCC incluye dos subprogramas referentes al control de autoridades: a) Name Authority Cooperative O perations (NACO), que revisa y actualiza la lista de autoridades LCNAF con más de 3.7 millones de registros de nombres de personas, entes corporativos, conferencias y lugares geográficos, así como títulos uniformes reunidos desde 1977, con unas 228.000 adiciones anuales y b) Subject Authority Cooperative Operations (SACO), que revisa y actualiza la lista de autoridades Library of Congress Subject Headings (LCSH), con más de 227.000 encabezamientos de materia o referencias y unas 9000 adiciones por año. El PCC incluye también un convenio particular entre British Library, National Library of Canada y Library of Congress, a partir del cual se está conformando un pod de autoridades en inglés, que se ha dado en llamar AngoAmmican Authority File(Library of Congress 1995b; Riemer y Morgenroth, 1993; Tabb, 1996; Tillett, 1995a,b y 1996).

Otras fuentes de control compartido de autoridades son las organizaciones como RLIN, WLN u OCLC. Esta última organización, que también participa del PCC, adquiere los registros de autoridades de Library of Congress, los somete a la revisión de sus bibliotecas cooperantes autorizadas por NACO que agregan referencias cruzadas; el registro de autoridades, así enriquecido sigue dos vías: por un lado vuelve a Library of Congress para su ingreso en las listas nacionales y por otro lado se registra en la lista de autoridades de OCLC, y queda disponible para ser copiado por cualquiera de las bibliotecas adheridas, las que a su vez pueden agregar nuevas referencias para su sistema local.

O tra experiencia interbibliotecaria importante es el proyecto AUTHOR, patrocinado por la Comisión Europea de Bibliotecas Nacionales (CENL), en el que 
participan la Bibliothèque National de Francia, la Bibliothèque Royale Albert I de Bélgica, el Instituto de Biblioteca Nacional e do Livro de Portugal, la Biblioteca Nacional de España y la British Library de Gran Bretaña. AUTHOR forma parte del proyecto Computerised Bibliographic Records Actions (COBRA) y se basa en los principios de cooperación internacional de IFLA (que se mencionan a continuación), se limita a nombres de personas y entes corporativos, pretende unificar los formatos nacionales en Unimard authorities y diseñar una red con acceso Z39.50 a la lista de autoridades (CENL, 1997; Jáudenes Casaubón, 1995).

Por su parte, IFLA ha propuesto que la cooperación intemacional para el control de autoridades se fundamente en dos principios: a) que cada país elabore su lista nacional de autoridades y b) que los demás países acepten las formas autorizadas por esas listas nacionales (D elsey, 1989; Tillet, 1989, 1995 y 1996; Willer, 1996). Aunque en teoría estos principios resultan lógicos, en la práctica presentan conflictos, ya que la autoridad establecida por un país puede no ser satisfactoria en otro, sea por el idioma, por la escritura, porque es más conocida una forma variante, etcétera. D esde Library of Congress se está impulsando una iniciativa que pretende cambiar el concepto de control de autoridades por el de control de acceso, en el que no habría una única forma autorizada, sino que distintas variantes tendrían el mismo valor; luego, si cada biblioteca local o cada sistema nacional lo desea, podría elegir una forma por default (Barnhart, 1996; Tillet, 1990, 1995 y 1996); sin embargo, consideramos que se requieren más experiencias para asegurar que esta modalidad no afecte la normalización. IFLA también ha propuesto la creación de un número estándar internacional (ISAD N) que identifique cada autoridad, para facilitar el intercambio entre distintas listas.

También es necesario establecer qué le compete al ámbito internacional, al nacional y al local, ya que las necesidades de un ámbito pueden diferir de los otros y obstaculizar la cooperación. De acuerdo con IFLA (D elsey, 1989), la agencia bibliográfica nacional es la responsable de compilar la lista nacional de autoridades, coordinando las bibliotecas locales en un sistema nacional y estableciendo mecanismos que aseguren la calidad y distribución de estas listas. A su vez, la agencia bibliográfica nacional puede acudir al ámbito internacional para intercambiar su lista nacional con las de otros países. La agencia bibliográfica nacional, entonces, pone estas listas de autoridades extranjeras a disposición de las bibliotecas locales de su país. Diversas naciones cuentan con sus listas de autoridades nacionales, entre ellas Alemania (Muennich, 1996), Australia (Trianor, 1995), Israel (Lazinger, 1991), Croacia (Willer, 1993), los países integrantes de AUTHOR, que incluyen a Portugal y España, cuyas listas resultan muy importantes para América Latina (CENL, 1997), Chile (RENIB, 1997), etcétera.

Al ámbito local le corresponde identificar las autoridades locales y transferirlas a la agencia bibliográfica del país para su inclusión en la lista nacional, copiar las autoridades que necesite a partir de las listas que la agencia bibliográfica nacional ponga a su disposición y diseñar el sistema local de autoridades en función de sus 
usuarios finales. En este ámbito son importantes las redes estatales o provinciales como ILLNET Online (Henigman, 1991).

En definitiva, hay tres factores importantes que no deben descuidarse si se quiere llegar a establecer un control bibliográfico universal (D elsey, 1989): a) la estandarización de prácticas para el establecimiento de encabezamientos y la estructuración de autoridades; b) la promoción de responsabilidades nacionales en la creación y diseminación de registros de autoridad y c) la planificación de una infraestructura que soporte el intercambio efectivo de datos de autoridad en la esfera internacional.

\section{EL CONTROL DE AUTORIDADES EN BIBLIOTECAS DE AMÉRICA LATINA}

El desarrollo de la informática entre las bibliotecas latinoamericanas ha dado lugar a situaciones dispares. En un intento por generalizar, podemos plantear que por un lado se encuentran aquellas bibliotecas que han incorporado programas de gestión bibliotecaria de origen estadunidense o europeo, los formatos tipo MARC y el acceso a los servicios de organizaciones como OCLC, RLIN, WLN, etcétera. Por otro lado, se encuentran las bibliotecas que utilizan sistemas de gestión bibliotecaria diseñados localmente, muchos de ellos basados en el software CDS/ ISIS para microcomputadoras (Microisis), que realizan procesos técnicos originales y no por copia y utilizan formatos no MARC, como los suministrados por organismos internacionales (CEPAL, AGRIS, INIS, LILACS, etcétera) o bien formatos locales como FOCAD en Argentina o FC en Uruguay.

Las bibliotecas del primer grupo son menos numerosas y se encuentran por supuesto en una situación de mayor ventaja para implementar el control de autoridades, en la medida que acceden a los recursos y desarrollos mencionados previamente en este texto. En cambio, para las bibliotecas del segundo grupo se presentan otros desafíos. Los formatos de descripción bibliográfica no MARC difundidos en la región no cuentan con sus respectivos formatos de autoridades, situación que genera enormes dudas respecto a si es conveniente esperar que aparezcan tales formatos o si es preferible anticiparse y definir las listas de autoridades con USMARC o UNIMARC. El tema es aún más preocupante si se tiene en cuenta que el formato que se elija para la lista de autoridades debe ser compatible con aquellos utilizados nacional e internacionalmente y al mismo tiempo con los catálogos locales a los que debe vincularse y que ya han sido definidos con otros formatos.

Los programas de gestión bibliotecaria diseñados en la región no han incluido hasta ahora el control de autoridades ni de nombres ni de materia y deberán evolucionar en este sentido, como ha comenzado a hacer SIABUC de la Universidad de Colima en México. Los desarrollos futuros deben tener en cuenta no sólo la implementación de las listas de autoridades, sino también la reconversión de lis- 
tas ya existentes (muchas de ellas implícitas) y la vinculación de estas autoridades nuevas con los puntos de acceso ya registrados en los catálogos y bibliografías en línea, que con seguridad requerirán correcciones a gran escala para coincidir con dichas autoridades.

En algunos países de la región, la biblioteca nacional ha organizado y coordina el sistema interbibliotecario nacional y proporciona a los participantes su lista de autoridades, como es el caso de Chile (RENIB, 1997), pero en otros países la biblioteca nacional se ha desentendido de la responsabilidad que le compete en cuanto a la cooperación, la normalización de formatos y procedimientos y la compilación de catálogos, bibliografías y listas de autoridades nacionales, evidenciando una vez más la carencia de políticas de información de los gobiernos respectivos. Numerosas bibliotecas especializadas han podido suplir esta falta de un formato nacional, participando en sistemas cooperativos coordinados por organismos internacionales, con lo cual -como ya se mencionó- se han incorporado, dentro de un mismo país, distintos formatos y metodologías de trabajo, situación que podría extenderse al control de autoridades.

En la región se han implementado sistemas para el control de autoridades como los informados por el Colegio de México y la Biblioteca de la Universidad Intercontinental (Moreno y Quijano Solís, 1991; Vela de la Sancha, 1996), así como la Red Bibliodata de Brasil; también se han compilado listas de autores personales e institucionales como la de CEPAL en Chile o las de la Universidad Nacional de San Juan y UNIRED en Argentina (Lohe, 1996) y de materia como la Lista deencabezamientos demateria para biblictecas compilada en Colombia o el Epigafianiode la Biblioteca Central de Ciencia y Técnica de Cuba, pero sería muy útil conocer con exactitud cuáles son las listas de autoridades existentes, las experiencias surgidas de esta compilación, el estado de compatibilidad que guardan unas con otras y las posibilidades de intercambio de registros de autoridades.

Por su parte, las escuelas latinoamericanas de bibliotecología deben incluir este tema en sus planes de estudio, formando a los futuros bibliotecarios en el uso de los formatos, manuales y directrices que normalizan el control de autoridades en línea; no obstante, es posible que en los años inmediatos estas escuelas deban centrar mayores esfuerzos en la educación continua o de posgrado, que permita la actualización y perfeccionamiento de los bibliotecarios en ejercicio.

\section{CONCLUSIONES}

La pregunta que nos planteamos en este momento es si vale la pena el control de autoridades, es decir, si los beneficios que aporta justifican los costos elevados. Las principales ventajas podrían resumirse en que a) mejora la precisión y la reall orientando al usuario durante la búsqueda a través del sistema de referencias 
cruzadas, b) en la recuperación le proporciona al usuario un 100\% de garantía documental (ninguna búsqueda con cero registros recuperados), c) mejora y agiliza los procesos técnicos y las operaciones de verificación, validación y cambios globales y d) ahorra espacio de almacenamiento en el catálogo en línea. Las desventajas estarían en el alto costo y el esfuerzo intensivo que demanda. En general, la comunidad bibliotecaria parece estar de acuerdo en que el control de autoridades es necesario, pero se necesitan cambios para hacerlo más eficiente (O ’Neill, 1996; Tillet, 1995 y 1996; Y ounger, 1995a,b).

El periodo 1996-2000 se presenta como crítico para el desarrollo del control de autoridades, según lo sugieren los planes estratégicos de OCLC (1995) y Library of Congress (1996b), que apuntan principalmente a lograr mejoras tecnológicas, aumento de la productividad y promoción de la cooperación. Se necesita además investigar a fondo la interacción entre los procesos técnicos y la recuperación para determinar dónde resulta más efectivo el control de autoridades, cómo se complementa éste con la recuperación palabra por palabra, las técnicas de búsqueda, la retroalimentación relevante, así como evaluar los programas de gestión y los costos (Taylor, 1989; Tillett, 1995 y 1996; Y ounger, 1995a,b).

Respecto a la región latinoamericana, consideramos que sería conveniente aprovechar que el control de autoridades en línea es un tema que se encuentra en su etapa inicial para a) dar a conocer las experiencias de implementación, gestión y evaluación de listas de autoridades, b) realizar una tarea cooperativa de normalización nacional y regional, c) promover la investigación y el desarrollo tecnológico y d) incluir este tema en los programas de enseñanza tanto de pregrado como de posgrado en las escuelas de bibliotecología.

No queremos dejar de insistir, a manera de cierre, en el compromiso que deben asumir las bibliotecas nacionales de América Latina, respecto a los beneficios que ofrece la cooperación interbibliotecaria en ésta como en otras tareas de procesos técnicos. Si sus contrapartes en los países desarrollados, como Library of Congress y las bibliotecas nacionales europeas, se han visto en la necesidad de cooperar, con mayor razón resulta imprescindible esta decisión entre las bibliotecas nacionales de países en vías de desarrollo -y por ende de escasos recursos-, ya que como afirma Tabb (1995), los procesos técnicos compartidos permiten alcanzar una mayor producción de registros, tanto de autoridad como bibliográficos, una calidad garantizada por el uso de normas y procedimientos comunes y periódicamente revisados, una eficacia basada en la sustancial reducción de la carga de trabajo, de los tiempos y de los costos, un ámbito ideal para que los bibliotecarios de procesos técnicos, liberados de las tareas rutinarias, dediquen sus esfuerzos a discutir la solución de problemas, se capaciten y capaciten a otros colegas, influyan decisivamente en el medio profesional mediante la normalización y recomendación de buenas prácticas bibliotecarias y exploren nuevas formas de acceso a la información. 


\section{BIBLIOG RAFÍA}

Aitchison, J., Gilchirst, A. (1987). Thesaunus constrution 2 ed. London: Aslib.

Aliprand, J. M. (1993). "Linking of alternate graphic representation in USMARC authority records". Catalogingand dassification quartely, 18(1):27-62.

Anderson, D . (1991). "Automatically generated reference in minimal-level authority records". Information techndogyandlibraries 10(4):251-62.

Bangalore, N.S. (1995). "Authority files in online catalogs revisited". Catalogingand dassification quartely; 20(3):75-94.

Barnhart, L. (1996). "Access control records: prospects and challenges". AuthorityContrd in the 21st Century: An Invitational Conference, March 31-April 1, 1996. Dublin, O hio: OCLC.

http:/ / www.oclc.org/ oclc/ man/ authconf/ confhome.htm

Bechtel, J.M. (1992). "An authority control alternative for small colleges”. Collegeandre searchlibranies 53(6):485-98.

Bogan, R. (1996). "Identifying personal name headings with cross references: a study to measure the effectiveness of selective authority control in a public library database". Authority Contrd in the21st Century: An Invitational Conference, March 31-April 1, 1996. Dublin, O hio: OCLC.

http:/ / www.oclc.org/ oclc/ man/ authconf/ confhome.htm

Borgman, C.L. (1996). "Why are online catalogs still hard to use?" Jamal of theAmeican Socidy for Informaion Saiene, 47(7):493-503.

Byrd, J., Sorury, K. (1993). "Cost analysis of NACO participation at Indiana University". Catalogingand dassification quartely; 16(2):107-23.

Calhoun, K. (1996). "Characteristics of member established headings in the OCLC database". Authority Contrd in the21st Century: An Invitational Conference, March 31-April 1, 1996. Dublin, O hio: OCLC.

http:/ / www.oclc.org/ oclc/ man/ authconf/ confhome.htm

Calhoun, K., O skins, M. (1992). "Rates and types of changes to LC authority files". Infomation techndogy andlibranies 11(2):132-6.

CENL (1997). COBRA: Compurtised bibliographicrecords actions

http:/ / portico.bl.uk/ gabriel/ en/ projects/ cobra.html

Clack, D.H. (1985). "Authority control: issues and answers”. Technical services quartety, 3(1/ 2):127-40.

- -. (1990). "Authority control and linked bibliographic databases". Catalogingand dassification quartely, 10(3):3-17.

D elsey, T. (1989). "Authority control in an international context". Catalogingand dassification quartely, 9(3):13-28.

Dickson, J., Zadner, P. (1989). "Authority control and the authority file: a functional evaluation of LCNAF on RLIN". Catalogingand dassification quartely; 9(3):57-73.

D ubois, C.P.R. (1987). "Free text vs. controlled vocabulary: a reassessment". Onlinere view 11(4):243-53.

Ensor, P. (1992). "User practices in keyword and boolean searching on an online public access catalog". Information techndogy andlibranies 11(3):210-9. 
Fidel, R. (1991). "Searchers'selection of search keys: III. Searching styles". Jaumal of theAmerican Sodiky of Infamation Saience, 42(7):515-27.

Fiegen, A.M.; Heitshu, S.C.; Miller, E.P. (1990). "The effect of the LASS microcomputer software on the cost of authority work in cataloging". Infomation techndogy andlibranies 9:253-7.

Fox, J.A. y Kanafani, K. (1989). "G lobal change capabilities to improve authority control in an online catalog". Information techndogy and libravies 8(3):273-83.

Fuller, E.E. (1989). "Variations in personal names in works represented in the catalog". Catalo g்ngand dassification quartely; 9(3):75-95.

Gibbs, G.E. y Bisom, D. (1989). "Creating an interactive authority file for names in the UCLA O rion system: specifications and decisions". Catalogingand dassification quartely; 9(3):153-69.

Goldman, H. y Havens, C. (1990). "A uthority control efficiency increase: NOTIS online authority and merged headings". Catalogingand dassification quartely, 12(2):27-45.

Goldman, H. y Smith, D.M. (1989). "Name authority in a NOTIS environment: Auburn University Libraries". Catalogingand dassification quartely, 9(3):121-31.

Gorman, M. y Winkler, P.W., editors (1988). AngoAmerican cataloging nules 2 ed rev. Ottawa: Canadian Library Association; Chicago: ALA.

Grady, A.M. (1996). "Practicalities of online authority maintenance: based on the University of Tennessee, Knoxville Libraries experience". Autharity Contrd in the21st Century: An Invitatio nal Conference, March 31-April 1, 1996. D ublin, O hio: OCLC. http:/ / www.oclc.org/ oclc/ man/ authconf/ confhome.htm

Halverson, J.A.; Gómez, J.; Marner, J.C. (1992). "Creation and implementation of an automated authority section at the Texas A\&M University Library". Catalogingand dassification quartely, 15(3):57-68.

Hancock-Beaulieu, M. (1990). "Evaluating the impact of an online library catalogue on subject searching behaviour at the catalogue and at the shelves". Jaumal of doumentation 46(4):31838.

Henigman, B. (1991). "Networking and authority control: online catalog authority control in Illinois". Infomation techndogyandlibraries 10:47-54.

Hunter, R.N. (1991). "Success and failures of patrons searching the on-line catalog at a large academic library: a transaction log analysis". Referencequartely; 30(3):399.

IFLA (1984). Guiddines for authority and reference entries London: IFLA International O ffice for UBC.

IFLA (1991). Unimard authorities Univesal fomat for authorities München: KG Saur (UBCIM publications: new series; 2).

IFLA (1993a). Direetrices para entradas deautoridades yreferenias Madrid: ANABAD, Arco/ Libro.

IFLA (1993b). Guiddines for subjet authrity and referenceentries München: KG Saur (UBCIM publications: new series; 12).

IFLA (1995). Direetrices para los reejstros de autoridad y referenia de materia Madrid: ANABAD, Arco/ Libro.

Irwin, D. (1993). "Local systems and authority control". Cataloging and dassification quartely, 16(2):55-69.

ISO 5127/ 3a (1981). Doumentation and information: Vocabulary: Part 3a. Acquisition, identification and analysis of douments and data. G eneva: ISO . 
Itoga, M.A.; Attig, J.; Avery, C. (1996). "Authority control profile of a large academic research library database". A uthority Contrd in the21st Century: An Invitational Conferen๕e March 31-April 1, 1996. Dublin, O hio: OCLC. http:/ / www.oclc.org/ oclc/ man/ authconf/ confhome.htm

Jáudenes Casaubón, M. (1996). “Control de autoridades: normalizar la normalización”. En: Megan Walls JA, coord. Tratadb básico de biblicteconomáa 2 ed. Madrid: Complutense, 1995.

Johnston, S.H. (1989). "Current offerings in automated authority control: a survey of vendors". Infomation techndogyandlibranies 8(3):236-64.

Kern-Simirenko, C. (1983). "OPAC user logs: implications for bibliographic instruction”. Libraryhigh techndog; 1(3):27-35.

Kirby, R.F. (1989). "Authority control in NOTIS". Cataloging and dassification quartely, 9(3):115-9.

Lancaster, F.W. (1995). Indizadón y resumen: teana y prádica Buenos Aires: E B Publicaciones.

Lazinger, S.S. (1991). "ALEPH: Israel's research library network: background, evolution, and implications for networking in a small country". Infomation techndogyandlibranies 10:275-91.

Library of Congress (1990). Library of Congess nule intepretations Washington DC: Library of Congress.

Library of Congress (1992). Subjet cataloging manual: dassification Washington DC: Library of Congress.

Library of Congress (1994). NACO participants' manual. Washington D C: Library of Congress.

Library of Congress (1995a). USMARC format for authority data induding guiddines for content designation Washington D C: Library of Congress.

Library of Congress (1995b). "History of the Program for Cooperative Cataloging: archive of early cooperative cataloging council reports". Washington DC: Library of Congess gopher:/ / marvel.loc.gov/ / cataloging/ coop/ coop_cncl

Library of Congress (1996a). Subjet catalogingmanual: subjet headings 5th ed. Washington DC: Library of Congress.

Library of Congress (1996b). The Program for Cooperative Cataloging five year strategic plan: 1996-2000. http:/ / lcweb.loc.gov/ catdir/ pcc/ stratplan.html

Lohe, R. (1996). "Información bibliográfica en diskette de la BD-UADE". SISBI, ABGRA. Enauntro Nacional deBiblictecas Universitarias, Buenos Aires, 16 y 17 de abril de 1996. Buenos Aires: SISBI, ABGRA.

Maccaferri, J.T. (1992). "Managing authority control in a retrospective conversion project”. Catalogingand dassification quartely, 14:145-67.

McCurley, H.H. (1993). "Implementation of an online series authority file at Auburn University". Catalogingand dassification quartely, 18(2):41-58.

McGrath, E. (1993). "D eveloping priorities for authority work at the University of Buffalo Libraries". Catalogingand dassification quartely; 16(4):5-23.

McJunkin, M.C. (1995). "Precision and recall in the keyword searches". Information te dndogyandlibranies 14:161-71. 
Millsap, L. y Ferl, T.E. (1993). "Search patterns of remote users: an analysis of OPAC transaction logs". Infomation techndogy and libranies 12(3):321-43.

Moreno Jiménez, M.P. (1992). XXIII Jomadas Mexicanas deBiblicteconomáa Universidad Autónoma de Yucatán, Mérida, 1992: El control de autoridad y los sistemas automatizados para bibliotecas: criterios de evaluación. México, D.F.: El Colegio de México.

Moreno, P.M. y Quijano Solís, A. (1991). V Cdoquio sobreAutomatizadón deBiblictecas Universidad de Colima, 1991: Los catálogos de autoridad de materia en el contexto automatizado. México, D.F.: El Colegio de México.

Muennich, M. (1996). "Authority control in Germany". Authority Contrd in the21st Century: An Invitational Conference, March 31-A pril 1, 1996. D ublin, O hio: OCLC. http:/ / www.oclc.org/ oclc/ man/ authconf/ confhome.htm

O 'Neill, E.T. (1996). "Introduction". Autharity Contrd in the21st Century: An Invitational Conferen@ March 31-April 1, 1996. Dublin, Ohio: OCLC. http:// www.oclc.org/ oclc/ man/ authconf/ confhome.htm

OCLC (1995). OCLC's automated authority contrd stratey, July, 1995. D ublin: OCLC. http:/ / www.oclc.org/ oclc/ man/ 9391 ausy/ aacs.htm

Olsen, R.J.; Christensen, J.O.; Larsen, K.A.; Willing, K. (1989). "Implementing NOTIS keyword/ boolean searching: a case study". Catalogingand dassification quartely, 10(2):125-40.

Park, A.L. (1992). "Automated authority control: making the transition". Speial libraries 83: 75-85.

Peters, T.A. (1989). "When smart people fail: an analysis of the transaction logs of an online public access catalog". Jaumal of acadamiclibranianship, 15(5):267-73.

RENIB (1997). ReelNacional deInfomaión Bibliogáfica. http:/ / www.dibam.renib.cl:80/ isc5.

Ridgeway, M. (1989). "Authority control on the GEAC Bibliographic Processing System". Catalogingand dassification quartely; $9(3): 133-52$.

Riemer, J.J. y Morgenroth K. (1993). "Hang together or hand separately: the cooperative authority work component of NACO". Catalogingand dassification quartely, 17(3/4):127-61.

Ríos García, Y. (1991). "Catálogos en línea de acceso público: selección bibliográfica”. Revista españda dedoamentacoón científica; 14(2):121-41.

Santizo, N.A. y Rezabek, C.S. (1992). "Series authority control: report of a survey". Cataloging and dassification quartely; 15(1):75-81.

Shiao-Feng, S. (1994). "Dialogue with an O PAC: how visionary was Swanson in 1964?” Library quartely; 64(2):130-61.

Sinnott, E. (1993). "Fewer errors resulting from the users' misconception of the OPAC in 1992 than a decade ago: a comparative study of no direct hits and zero hits in author searches". Catalogingand dassification quartely; 18(1):75-101.

Sullivan, E. (1992). "Authority work on INNO PAC at Y ale Law Library". Tehnical sevicesquarterly9(4):43-8.

Svenonius, E. (1986). "Unanswered questions in the design of controlled vocabularies”. Jaumal of theAmerican Sociey for Information Saience 37(5):331-40.

Tabb, W. (1996). "The Program for Cooperative Cataloging: mission, goals, and potential for international cooperation: paper presented at the Cataloging Section O pen Program", IFLA Conference, Beijing, China, August 27th, 1996). Washington DC: Library of Congress. http:/ / lcweb.loc.gov/ catdir/ pcc/ tabbpaper.html 
Taylor, A.G . (1989). "Research and theoretical considerations in authority control". Ca talogingand dassification quartely; 9(3):29-56.

- - (1992). Bdhdan S. Wynar. Introduction tocatalogingand dassification 8 ed. Englewood: Libraries Unlimited:472-90.

Tillett, B.B. (1989). "Considerations for authority control in the online environment". Catalogingand dassification quartely; 9(3):1-11.

- - . (1990). "Access control: a model for descriptive, holding and control records". G orman M, editor. Convergence Procedings of the Seeond National Conference of theLibrary and Infomation Tehndogy Association O ctober 2-6, 1988, Boston. Chicago: ALA (Library and information technology series; 3).

- -. (1995). "21st Century authority control: what is it and how do we get there?" The Future is Now. Reconiling Change and Continity in Authority Contrd: Proceedings of the OCLC Symposium ALA Annual Conference, June 23, 1995. Dublin, Ohio: OCLC. http:/ / www.oclc.org./ oclc/ man/ 9391ausy/ toc.htm

- - . (1996). "International shared resource file". Authority Contrd in the21st Century: An Invitational Conference March 31-April 1, 1996. Dublin, Ohio: OCLC. http:/ / www.oclc.org/ oclc/ man/ authconf/ confhome.htm

Tillotson, J. (1995). "Is keyword searching the answer?" College and rearch libraries 56:199-206.

Trainor, J. (1995). "ABN Standards Committee: Authority control in the NDIS". Canbe ma: National Library of Australia http:/ / www.nla.gov.au/ 2/ ...mittees/ agencia bibliográfica nacionalsc 199515.html

UNE 50113/ 3a (1991). Infomadóny doamentadón: V oabularia Parte3a): Adquisiaón, identificadón yanálisis dedbamentosy datos Madrid: AENOR.

Van Pulis, N. (1996). "Authority records: characteristics of change". AuthorityContrd in the 21st Century: An Invitational Conference March 31-April 1, 1996. Dublin, O hio: OCLC. http:/ / www.oclc.org/ oclc/ man/ authconf/ confhome.htm

Vela de la Sancha, J. (1996). “El catálogo de autoridad de materias de la Biblioteca de la Universidad Intercontinental”. XXV II Jomadas Mexicanas de Biblicteconomía, O axaca, 1996.

Watson, M.R. y Taylor, A.G. (1987). "Implications of current reference structures for authority work in online environments". Infomation techndogyandlibraies 6(1):10-9.

Wendler, R. (1995). "Automating heading correction in a large file: Harvard's experience". TheFutureis Now. ReconilingChangeand Continuityin A uthority Contrd: Proceedings of theOCLC Symposium ALA Annual Conference, June 23, 1995. Dublin, O hio: OCLC. http:/ / www.oclc.org./ oclc/ man/ 9391ausy/ toc. htm

Wilkes, A. y Nelson, A. (1995). "Subject searching in two online catalogs: authority control vs. non-authority control". Catalogingand dassification quartely, 20(4):57-79.

Willer, M. (1993). "The implementation of UNIMARC/ authorities in the Croatian Library Information System”. Intemational Catalogingandbibliographiccontrd; 22(4):64-7.

- - . (1996). "Authority control and international standard authority data numbers: need for international cooperation". Authority Contrd in the21st Century: AnInvitational Conference March 31-April 1, 1996. Dublin, O hio: OCLC.

http:/ / www.oclc.org/ oclc/ man/ authconf/ confhome.htm 
Younger, J.A. (1995a). "After Cutter: authority control in the twenty-first century". Libraryre sarres and technical services 39(2):133-41.

- - . (1995b). "Reframing the authority control debate". TheFutureis Now ReconilingChangeand Continuity in Authority Contrd: Procedings of theOCLC Symposium ALA Annual Conference, June 23, 1995. D ublin, O hio: OCLC.

http:/ / www.oclc.org./ oclc/ man/ 9391ausy/ toc.htm

- - . (1996). "Access standards and retrieval". Authority Contrd in the21st Century: An Invitational Conference, March 31-A pril 1, 1996. Dublin, O hio: OCLC.

http:/ / www.oclc.org/ oclc/ man/ authconf/ confhome.htm

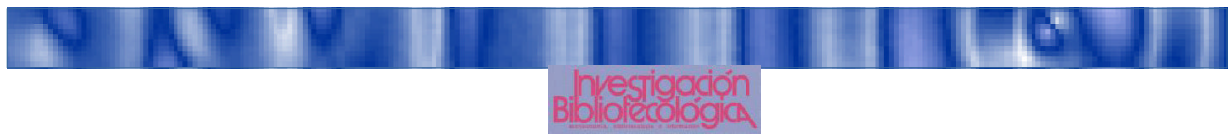

